\title{
Mobile Multicast with Routing Optimization for Recipient Mobility ${ }^{1}$
}

\author{
Jiunn-Ru Lai, Wanjiun Liao, Ming-Yu Jiang and Chien-An Ke \\ Department of Electrical Engineering \\ National Taiwan University \\ Taipei, Taiwan \\ Email:wjliao@cc.ee.ntu.edu.tw
}

\begin{abstract}
This paper explores the issues of multicasting for mobile hosts using IETF Mobile IP. Mobile IP suggests two approaches for mobile multicasting, namely, remote subscription (MIP-RS) and bi-directional tunneling (MIP-BT). MIP-RS uses a nä̈e join-and-leave policy for mobile hosts and causes serious packet losses while roaming. MIP-BT is free from packet losses due to roaming; however, MIP-BT and its modification MoM suffer from inefficient routing and wastage of system resources. In this paper, we propose a new mobile multicasting protocol called Mobile Multicast with Routing OPtimization (MMROP) that employs a modified join-and-leave mechanism, and routes the missing data sequence due to the "out-of-synch" problem to other agents in the adjacent networks via tunneling for roaming terminals. With MMROP, the mobility agent in Mobile IP is extended to assist multicasting for mobiles. We also developed a cost analytical model to evaluate the performance of MMROP, in comparison to MIP-RS, MIP-BT, and MoM. The results demonstrate that MMROP has optimal routing efficiency, low delivery cost and high robustness, as compared to other approaches.
\end{abstract}

\section{INTRUDUCTION}

Having been an important subject both in research and development [1][2], IP multicast may be used in many cxisting and emerging Internet applications. A multicast data packet contains a class $\mathrm{D}$ group address in the destination address field of the IP header, being delivered to destination group members with the same unreliable and best effort service as in unicast IP data transmission. It is not necessary for a host to join a group in order to send datagrams to the group members. Individual hosts are free to join or leave a multicast group at any time, without affecting other group members. IP multicast is cooperatively implemented through two mechanisms: local group management (such as IGMP [3] and RGMP [4]) and global multicast routing.

While existing work [5-10] has investigated IP multicasting for mobile hosts using different mobile technologies, this paper, in particular, attempts to study the problem of mobile multicast using IETF Mobile IP [11], the dominant standard for IP mobility. Mobile IP defines two approaches of mobile multicast to accommodate the join and leave dynamics in group participation and location change for roaming terminals: remote subscription (MIP-RS) and bi-directional tunneling (MIP-BT). Remote subscription requires a mobile to resubscribe to the joined group on the newly visited foreign network while roaming. While having the advantages of simple implementation and optimal routing efficiency, MIPRS suffers from serious packet losses due to using naike joinand-leave mechanism for mobiles to participate in the groups of interest. As a result, it causes long join and graft latency to participate in the interested group when there is no other group members in the newly visited network. In addition, due to network dynamics in packet delivery, even if there are other participants in the joined group in the newly visited network, MIP-RS still has the "out-of-synch" problem. With the bidirectional tunneled multicast, a mobile must subscribe to the groups of interest through the home agent. When the mobile is away from home, the multicast datagrams are first routed to the home agent, from where the datagrams are encapsulated in a unicast packet destined to the mobile via tunneling. Thus, MIP-BT suffers from the inefficient delivery of triangle routing and low scalability to accommodate the large number of mobile terminals. Mobile Multicast Protocol (MoM) [8,9] attempts to enhance the scalability of MIP-BT by the Designated Multicast Service Provider (DMSP) to solve the tunneling convergence problem of MIP-BT. While improving the scalability as compared to MIP-BT to some extent, MoM, however, still suffers from the triangle routing problem since it is based on MIP-BT. Besides, MoM does not promise nolosses from roaming as in MIP-BT, and suffers from the outof-synch problem when mobiles roam from one subnet to another.

In this paper, we propose a new protocol called Mobile Multicast with Routing OPtimization (MMROP) for mobile multicasting. Unlike MoM based on MIP-BT, MMROP is derived from MIP-RS, and with the assistance of mobility agents, routes the missing datagrams due to the out-of-synch problem in adjacent subnets via tunneling to ensure optimal routing efficiency and no packet losses from roaming. We also develop a simplified cost analytical model to evaluate the performance of the four approaches. The results consistently demonstrate that MMROP enjoys optimal routing efficiency as in MIP-RS, high robustness while roaming as in MIP-BT, and high performance in terms of datagram delivery cost as compared to the other three approaches.

The rest of the paper is organized as follows. The issues of IETF Mobile IP multicast are addressed in Section II. The MMROP operations are described in Section III. The performance comparison of MMROP with MIP-RS, MIP-BT, 
and MoM is presented in Section IV, including cost analysis. Finally the concluding remarks are included in Section V.

\section{IETF Mobile IP: FROM UNICAST TO MULTICAST}

While Mobile IP offers two approaches for multicasting, namely, MIP-RS and MIP-BT, both approaches suffer from problems when used as a mobile multicast mechanism. MIPRS suffers from packet losses from roaming due to using the nä̈e join-and-leave policy, causing poor service quality of multicasting for mobiles as compared to stationary hosts. MIP-BT allows no packet losses from roaming, because multicast datagrams are first routed to the home agent in the home network and from where the packets are tunneled to mobiles in the visited foreign networks. However, strictly speaking, MIP-BT (and its modification MoM) should not be considered as a multicast mechanism, but unicast emulation for multicast, because using MIP-BT to handle mobile multicast is just like using multiple unicast transmissions to simulate one multicast transmission. Recall that multicast is a one-to-many or many-to-many communication scenario, which duplicates multicast datagram only if necessary. Fig. 1. (a) depicts the difference of multicast from using multiple unicast delivery to simulate one multicast transmission. Fig. 1. (b) is a comparison of MIP-RS with MIP-BT, and the difference is similar to that in Fig. 1 (a). Thus, in terms of data transmission and resource usage, MIP-RS is a better candidate for mobile multicast as compared to MIP-BT.

To extend IP unicast to multicast in 1989, [3] introduced a

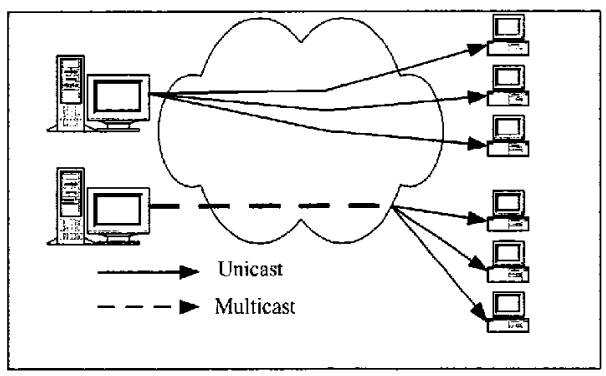

(a) Multicast vs. unicast

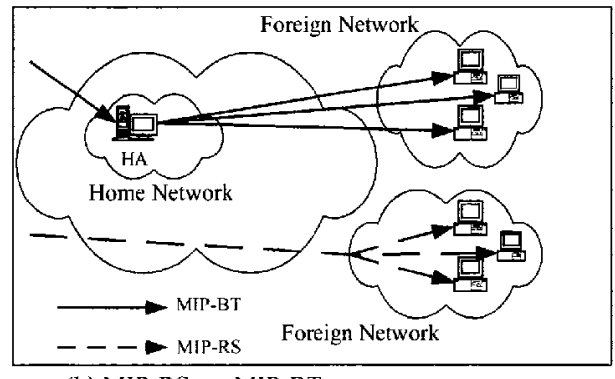

(b) MIP-RS vs. MIP-BT

Fig. 1. Datagram delivery group management mechanism called Internet Group Management Protocol (IGMP). Extending Mobile IP unicast to multicast is not as trivial as in MIP-RS, which allows mobiles using IGMP to report membership directly to multicast routers and results in poor service quality of multicast, because IGMP is designed to support static hosts. Thus, under the condition of no change in the existing group model, to extend Mobile IP unicast to multicast, some mechanism between the multicast routers and mobile hosts must be introduced. In the following section, we will describe our approach called MMROP for mobile multicast, extending the mobility agent in Mobile IP to be the proxy of multicast services for mobile hosts, taking advantage of the static nature of the mobility agent.

\section{MOBILE MULTICAST WITH ROUTING OPTIMIZATION (MMROP)}

This section describes MMROP in details. In the following, we will focus only on recipient mobility. Sender mobility in MMROP can be supported simply by sending multicast datagrams from a mobile in the foreign network back to the home agents in the home network via tunneling, as in MIP-BT.

\section{A. The Characteristics of MMROP}

MMROP is derived from MIP-RS and thus enjoys the advantages of high routing efficiency as in MIP-RS. The system components and the operation of Mobile IP in unicast delivery remain unchanged. Mobility management is still cooperatively supported through a home agent and foreign agents via tunneling and mobility binding. While not necessarily a multicast router, the mobility agent, in addition to mobility management, also serves as a proxy of multicast services for mobiles. From the perspective of a multicast router, the agent is just like a group participant, and from mobiles, the agent enables multicast service. Thus, the mobility agent must join the multicast group of interest on behalf of the mobiles in its affiliated subnet.

The table maintained by the mobility agent is extended to address group information, including a buffer (cache), a serving list and a tunneling list, on a per-group basis. The cache of a group, say, G, temporarily stores the multicast datagrams destined for group $\mathrm{G}$ in the affiliated network. The serving list maintains the registered mobiles participating in group $G$ in the network. The tunneling list records the mobile hosts previously registered with this agent but are currently roaming to adjacent networks managed by other foreign agents and requesting to recover the lost datagrams due to the out-ofsynch problem. The mobility agent encapsulates the datagrams requested by the mobiles in the tunneling list from the cache in unicast packets to the corresponding foreign agents in the adjacent networks, where the tunneled packets are decapsulated, stored in the corresponding cache entries of group $\mathrm{G}$ and forwarded to the affiliated networks. 


\section{B. Operation Overview for $M M R O P$}

When a mobile host $(M H)$ roams to a visited network, say subnetl, the mobile is first registered with the foreign agent $F A 1$, as in Mobile IP unicast. If the mobile would like to join a multicast group, say group $\mathrm{G}$, the mobile issues a join ${ }^{2}$ message for group G to $F A 1 . F A l$ then sends an IGMP-join message for the multicast group $G$ to the immediately neighboring multicast router on behalf of the mobile if there is no other mobiles participating in group $\mathrm{G}$ in subnet1. Upon joining group $\mathrm{G}, F A l$ creates a cache entry, temporarily storing data received from group G. $F A I$ adds $M H$ to the serving list of multicast for group $\mathrm{G}$.

When the mobile is roaming across service boundary (say from subnet1 to subnet2), a handoff is performed so as to ensure multicast datagrams are correctly received by $M H$. The mobile is registered with the new foreign agent $F A 2$, as in Mobile IP unicast. In addition, the mobile sends a join message for group $\mathrm{G}$ to $F A 2$. Again, $F A 2$ sends an IGMP-join message for group $\mathrm{G}$ to the immediately neighboring multicast router on behalf of the mobile $M H$ if there are no other mobiles participating in group $G$ in subnet2. Upon joining group $G$, $F A 2$ creates a cache entry, temporarily storing data received from group G. $F A 2$ adds $M H$ to its serving list of group G. Upon receiving a new packet in subnet2, the mobile checks if there is any offset in datagram sequence number from subnet2 to subnetl. If the packets received in subnet 2 are behind (in terms of sequence number) subnet 1 due to network dynamics, the mobile sends a leave message with zero offset to the old agent $F A 1$. Otherwise, the mobile will request $F A 2$ to transmit the offset from its cache. If $F A 2$ can recover the requested packets from its cache, the mobile sends $F A l$ a leave message with zero offset; otherwise, $M H$ sends $F A I$ the leave message with an offset block, say $[a, b]$, to be tunneled from $F A 1$ to $F A 2$, where the offset block is the difference in data sequence to be recovered between two adjacent subnets. On receipt of a tunneled packet from $F A 1$, if the encapsulated packet is detected as a multicast datagram of group $\mathrm{G}, F A 2$ will first store the multicast datagram in the cache entry of group $\mathrm{G}$ and then forward the datagram to its affiliated subnet. In this way, MMROP can solve the out-of-synch problem encountered by roaming hosts and supports cascading handoff scenarios in which the mobile moves to another subnet, say subnet3, while receiving the data for the offset block from $F A 1$.

On receipt of a leave message for group $G$ from a mobile host, if the leave message carries a non-zero offset block $[a, b]$, $F A /$ removes the host from the serving list to the tunneling list in the entry of group $\mathrm{G}$; otherwise, $F A l$ just deletes the mobile from the serving list. If $M H$ is moved to the tunneling list with offset block $[a, b], F A I$ tunnels data from $a$ to $b^{3}$ in the cache to

\footnotetext{
${ }^{2}$ which may or may not be an IGMP-Join report.

${ }^{3}$ As soon as the leave message is received, some packets up to $b$ may not have
}

FA2. FAl removes $M H$ from the tunneling list after the transmission has finished. Once both the serving list and the tunneling list are empty, $F A I$ deletes the cache of group $\mathrm{G}$ and issues an IGMP-leave message to the multicast router for leaving the group.

\section{PERFORMANCE EVAULATION}

\section{A. Cost Analysis}

We have developed a simplified analytical model of average cost per mobile for each subnet visited to compare the four approaches of mobile multicasting. Cost consists of two components: one is the processing cost, measured in time, and the other is the transmission cost, measured in bit-hop, i.e., it is the length of transmission in bits multiplied by the number of hops traversed. Fig. 2. shows the system architecture used by the model, the topology of which is extended from [12] which is used to evaluate the performance of Mobile IP Location Register in comparison to Mobile IP. The distance between two nodes is expressed as the number of hops in between (i.e., hop count), and indicated by $a, b, c$, and $d$.

Assume that the source generates multicast datagrams at a rate of $\lambda$, and a mobile moves from one subnet to another at a rate of $\mu$. The Packet to Mobility Ratio (PMR) [12] is defined as $P M R=\lambda / \mu$, which specifies the mean number of multicast datagrams received by a mobile host per move. The larger the $P M R$, the slower the speed at which the mobiles are moving across subnets and the longer the time the mobiles are staying in subnets. Parameters are summarized as follows.

\section{1) System parameters:}

$\lambda:$ the mean rate that a source generates multicast datagrams $\mu$ : the mean rate that a mobile moves from one subnet to another

$p:$ packet to mobility ratio ( $P M \mathrm{R})$, defined as $\lambda \mu$,

$q_{n \omega_{-} M}:$ probability that a mobile visits a subnet with no other members $\left(q_{n o_{-} M}=0.01\right)$

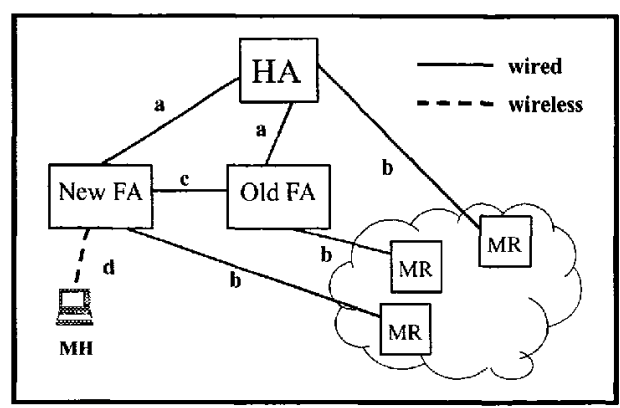

Fig. 2. The system architecture of the cost analytical model

been stored in the cache due to not having been received in this subnet. F $\Lambda$ will keep tunneling upon receipt of datagrams within the offset block. 
$q_{D M S P}:$ probability that a DMSP handoff is performed $\left(q_{\text {DMSP }}=0.05\right)$

$N$ : the average number of mobiles per group in a cell $(N=5)$ $n$ : the mean data offset in the adjacent subnets $(n=2)$

\section{2) Cost variables:}

$l:$ the ratio of the length of a datagram to the length of an ICMP control packet (assume a control packet is of length 1)

$r$ : the cost to process a packet in each node (assumed also for a control packet transmission cost for single hop, $r=1$ )

$C_{\text {conrrol }}$ : the cost to process control packets per handoff, including mobility binding and group participation

$C_{\text {datagram }}$ the cost to deliver multicast datagrams to a mobile in the newly visited subnet

$C_{H_{-} T}$ the cost to forward one multicast datagram from $\overline{\mathrm{H}} \mathrm{A}$ to a mobile via tunneling

$C_{F A T}$ the cost to forward one multicast datagram from $\mathrm{FA}$ to a mobile via tunneling

$C_{M R}$ the cost to forward one multicast datagram from the multicast router to a mobile

Without loss of generality, assume that each unit of processing cost is equivalent to $f$ units of transmission cost. For the system shown in Fig. 2., the average cost per mobile for each subnet visited (in transmission cost units) to enable mobile multicast for each approach is expressed as

$C_{\text {protocol }}=C_{\text {control }}+C_{\text {datagram }}$

where $C_{\text {comtrol }}$ and $C_{\text {clutagran }}$ are defined as follows:

(a) $C_{\text {controt }}=$ Mobile IP registration overhead + group participation overhead per handoff

$=2(a+d)+5 r f+\left\{\begin{array}{lr}0 & \cdots \mathrm{MIP}-\mathrm{BT} \\ q_{n o_{-} M}(b+d+2 r f) & \cdots \mathrm{MP}-\mathrm{RS} \\ q_{n \sigma_{-} M}(b+d+2 r f)+q_{D M S P}(2 c+3 r f) & \cdots \mathrm{MoM} \\ q_{n o_{-} M}(b+d+2 r f)+(2 c+3 r f)+\frac{n^{*} l}{N} \cdots \mathrm{MMROP}\end{array}\right.$

where $2(a+d)+5 r f$ is the cost for Mobile IP registration.

(b) $C_{\text {datugram }}=$ (datagram generation rate) $\times$ (mean sojourn time to stay in a subnet for a mobile) $\times$ (the cost to deliver a multicast datagram to a mobile in the newly visited network)

$$
\begin{aligned}
= \begin{cases}\lambda * \frac{1}{\mu} * \frac{C_{M R}}{N} & \cdots \mathrm{MIP}-\mathrm{RS} \text { and MMROP } \\
\lambda * \frac{1}{\mu} * \frac{C_{F A_{-} T}}{N} & \cdots \mathrm{MOM} \\
\lambda * \frac{1}{\mu} * C_{H A_{-} T} & \cdots \mathrm{MIP}-\mathrm{BT}\end{cases} \\
= \begin{cases}p * \frac{[l *(b+d)]}{N} & \cdots \mathrm{MIP}-\mathrm{RS} \text { and MMROP } \\
p * \frac{[l *(b+c+d)+2 * r f]}{N} & \cdots \mathrm{MoM} \\
p *[l *(b+c+d)+2 * r f] & \cdots \mathrm{MIP}-\mathrm{BT}\end{cases}
\end{aligned}
$$

Fig. 3. depicts the cost comparison of the four approaches using the MIP-BT as the basis, varying the $P M R$ value from 1 to 60 . Here we assume $f=1$. The curves are plotted by using the ratio of $C_{\text {protocol }}$ to $C_{M I P-B T}$, where ' $X$ ' is for $C_{M o M}$ to $C_{M I P-B T}$, ' $\mathrm{O}$ ' is for $C_{M I P-R S}$ to $C_{M I P-B T}$ and ' $*$ ' is for $C_{M M R O P}$ to $C_{M I P-B T}$. The lower the curve, the better the performance. In Fig. 3., the cost ratio of all three curves is less than 1, indicating that MIP-BT has the worst performance. Besides, of the other three approaches, since the curve of MIP-RS to MIP-BT is the lowest, followed by that of MMROP to MIP-BT, and finally by that of MoM to MIP-BT, MIP-RS has the best performance, MMROP is the second best, and MoM is the worst. MIP-BT performs the worst due to using unicast emulation for multicast and suffering from triangle routing and tunneling convergence. MoM enhances MIP-BT to some extent, eliminating the tunneling convergence problem from MIP-BT. However, MoM still suffers from the problems of triangle routing and tunneling overhead in packet transmission, since it is based on MIP-BT. MIP-RS has the lowest delivery cost, at the expense of high packet losses caused by the out-of-synch problem. The $P R M$ value reflects how fast the mobiles move. The larger the $P M R$ value, the longer the mobiles stay in subnets. Thus, as $P M R$ increases, all the curves become flat, thanks to the relatively non-mobile nodes acting like stationary hosts. The curves of MMROP stay very closely to that of MIP-RS, especially when $P M R$ becomes large.

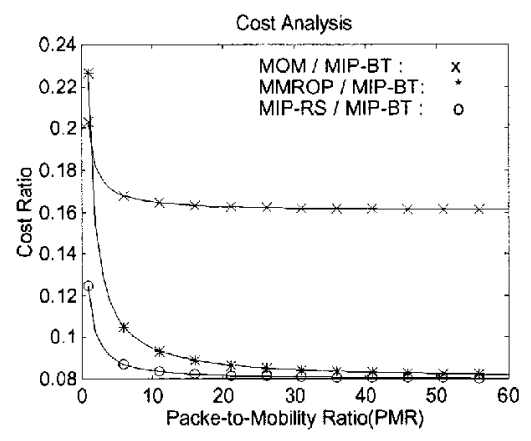

(a) Short datagrams, with $l=50$

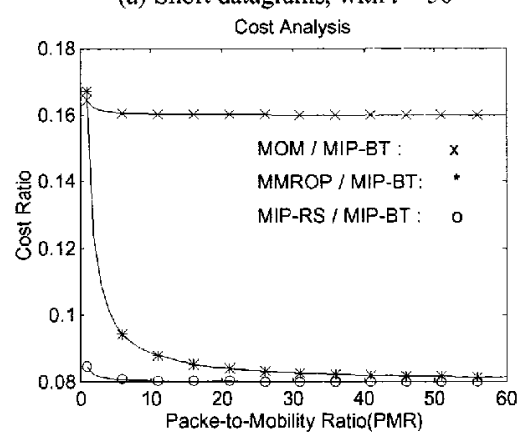

(b) Long datagrams, with $l=500$

Fig. 3. Cost comparisons of the four approaches for

$$
a=3, b=1, c=2, d=1, r=1, f=1
$$




\section{CONCULSION REMARKS}

In this paper, we have proposed a new protocol called Mobile Multicast with Routing OPtimization (MMROP) with the characteristics of optimal routing efficiency, robustness, scalability, simplicity, and flexibility to be a mobile multicast mechanism. With MMROP, the mobility agent in Mobile IP, in addition to mobility management, is extended to assist multicasting for mobiles. The mobility agent is not necessarily a multicast router. From the perspective of the multicast router, the mobility agent is a group participant, and from the mobiles, the agent serves as the proxy of multicast services. Unlike MoM based on MIP-BT, MMROP is derived from MIP-RS, and with the assistance of mobility agents, routes the missing datagrams due to the out-of-synch problem in adjacent subnets via tunneling to ensure optimal routing efficiency and no packet losses from roaming. We also developed a cost analytical model to evaluate the performance of MMROP, in comparison to MIP-RS, MIP-BT, and MoM. The results demonstrate that MMROP has optimal routing efficiency, low delivery cost and high robustness, as compared to other approaches.

\section{REFERENCES}

[1] Special issue on networked support for multipoint communications, IEEE JSAC, vol. 15, edited by M. $\mathrm{H}$. Ammar, G. Polyzos, and S. Tripathi, April 1997.

[2] J. C. Pasquale, G. C. Polyzos, and Xylomenos. "The Multimedia Multicasting Problem," ACM Multimedia Systems, vol. 6, 1998, pp. 43-59.

[3] S. Deering. "Host Extensions for IP Multicasting," IETF RFC 1112, Aug. 1989.

[4] W. J. Liao and D. N. Yang. "Receiver-initiated Group Membership Protocol," Proc. IEEE ICNP 99, Toronto, Canada, Nov. 1999.

[5] A. Acharya, A. Bakre and B. Badrinath, "IP Multicast Extensions for Mobile Internetworking," Proc. IEEE INFOCOM 96, San Francisco, CA, Mar. 1996

[6] A. Acharya and B. Badrinath. "Delivering Multicast Messages in Networks with Mobile Hosts," Proc. 13th Int. Conf. Distributed Computting Systems, Pittsburgh, PA, pp. 292-299, May 1993

[7] K. Brown and S. Singh, "RelM: Reliable multicast for mobile networks," Technical Report, Department of Computer Science, University of South Carolina, Sept. 1995

[8] V. Chikarmane et al. "Multicast Support for Mobile Hosts Using Mobile IP: Design Issues and Proposed Architecture," ACM/Baltzer Mobile Networks and Applications, v.3 no.4, pp.365-379, Jan. 1999

[9] T. G. Harrison et al. "Mobile Multicast (MoM) Protocol: Multicast Support for Mobile Hosts," Proc. ACM MobiCom 97, Budapest, Hungary, pp. 151-160, Sep. 1997.

[10] C. L. Williamson et al. "Performance Evaluation of the MoM Mobile Multicast Protocol," ACM/Baltzer Mobile Networks and Applications, v.3 no.2, pp. 189 - 201, Aug. 1998

[11] C. Perkins, "IP Mobility Support," RFC 2002, Mobile IP Working Group, Oct. 1996
[12] R. Jain et al. "Mobile Internet Access and QoS Guarantees Using Mobile IP and RSVP with Location Registers," Proc. IEEE ICC 98. 